\title{
Body Mass Index and Clinical Response to Tocilizumab in Patients With Rheumatoid Arthritis
}

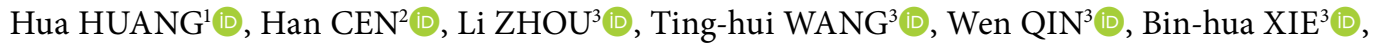 \\ Dong-Mei $\mathrm{XIAO}^{3} \mathbb{B}$, Xiu-Di WU ${ }^{3}$, Hua-Xiang WU' ${ }^{1}$ \\ ${ }^{1}$ Department of Rheumatology, Second Affiliated Hospital, School of Medicine, Zhejiang University, Hangzhou, China \\ ${ }^{2}$ Department of Preventive Medicine, Medical School of Ningbo University, Ningbo, China \\ ${ }^{3}$ Department of Rheumatology, Ningbo First Hospital, Ningbo Hospital of Zhejiang University, Ningbo, China
}

\begin{abstract}
Objectives: This study aims to determine whether baseline body mass index (BMI) affects clinical response to tocilizumab (TCZ) after six months of treatment in rheumatoid arthritis (RA) patients.

Patients and methods: In this prospective study, a total of 52 RA patients (10 males, 42 females; mean age $50.6 \pm 12.2$ years; range, 23 to 73 years) receiving intravenous TCZ were consecutively recruited and followed-up for six months. BMI was calculated before initiation of TCZ treatment. The primary clinical response criterion was clinical disease activity index (CDAI) low disease activity (LDA) and the secondary clinical response criteria included CDAI remission, disease activity score based on 28 joints (DAS28)-erythrocyte sedimentation rate (ESR) LDA, DAS28-ESR remission, European League Against Rheumatism (EULAR) good response, and decreased DAS28-ESR ( $\triangle D A S 28-E S R) \geq 1.2$.

Results: The number of RA patients classified as normal weight, overweight, and obese according to baseline BMI was 38 (73.1\%), eight (15.4\%), and six (11.5\%), respectively. Similar baseline BMI median levels were found between RA patients reaching CDAI LDA and non-LDA: 21.11 (18.94-23.72) versus $20.78(20.03-22.29)(p=0.98)$, and non-significant difference in the proportion of responders between normal weight and overweight/obese RA patients was found $(p=0.47)$. No significant difference was found when the secondary clinical response criteria were applied.

Conclusion: Our study demonstrates that BMI is not associated with clinical response to TCZ among RA patients and TCZ may be used to treat RA patients regardless of BMI levels.

Keywords: Body mass index, response, rheumatoid arthritis, tocilizumab.
\end{abstract}

Rheumatoid arthritis (RA) is a progressive, chronic, and relatively common autoimmune disease which is characterized by synovitis and the production of auto-antibodies. ${ }^{1}$ During the past decade, some innovative discoveries have been made in the pathogenic mechanism of RA, leading to the development of more and more novel biological disease-modifying anti-rheumatic drugs (bDMARDs). Currently approved bDMARDs for RA treatment include tumor necrosis factoralpha (TNF- $\alpha$ ) inhibitor, T-cell co-stimulation inhibitor, B-cell depletion and interleukin-6 (IL-6) receptor inhibitor, which have greatly improved the prognosis of RA patients. ${ }^{2}$

Tocilizumab (TCZ) is a recombinant humanized anti-IL-6 receptor monoclonal antibody which blocks the biological functions of IL- 6 from binding to its soluble and membrane-bound IL-6 receptor, which has been recommended as a firstline bDMARD for RA patients. ${ }^{3-5}$ However, not all RA patients could achieve treatment target under

Received: August 16, 2018 Accepted: February 01, 2019 Published online: April 22, 2019

Correspondence: Hua-Xiang Wu, MD. Department of Rheumatology, Second Affiliated Hospital, School of Medicine, Zhejiang University, 88 Jiefang Road, Hangzhou, Zhejiang, 310009, PR China. Tel: +86 571-89713754 e-mail: wuhx8855@sina.com 
TCZ treatment, indicating a significant proportion of RA patients who do not respond well to TCZ. Thus, identifying predictors of clinical response to TCZ would improve care in selecting patients who would be able to respond well.

Recently, the relationship between body mass index (BMI) and clinical response to bDMARDs in RA patients has attracted extensive attention. The rational for investigating the effect of obesity/ overweight on clinical response to bDMARDs is as follows: First, RA is a progressive disease. If disease activity is not controlled, irreversible damage would ensure. Second, the cost of bDMARDs is relatively high and adverse events might occur during treatment. Since several types of bDMARDs are available, if obese/ overweight RA patients do not respond well to some bDMARDs, the other bDMARDs may be selected in advance. Finally, pharmacokinetic variables such as drug clearance and volume of distribution may be influenced by overweight and obesity. The available studies indicate that BMI might hold the potential to guide the personalized treatment for infliximab (IFX), a TNF- $\alpha$ inhibitor. ${ }^{6-10}$ Whether BMI may also be used to guide the personalized treatment of other bDMARDs, particularly for TCZ, is of great interest, due to the relationship between IL- 6 and obesity. It has been suggested that IL-6 could exert significant effect on weight status. ${ }^{11-13}$ Spontaneous obesity could develop in mice lacking IL-6 gene, ${ }^{12}$ and weight gain was noted among a proportion of RA patients treated by TCZ.11,13 In addition, IL- 6 could be produced by adipose tissue or up-regulated by adipokines. ${ }^{14}$ Thus, it is reasonable to speculate if $\mathrm{BMI}$ influences the clinical response to $\mathrm{TCZ}$.

So far, only three studies have been performed to evaluate whether baseline BMI accounts for the inter-individual variance of clinical response to $\mathrm{TCZ}$ among RA patients, whereas non-significant evidence was found.11,15,16 Since sampling error is inevitable, the same topic should be evaluated multiple times to reach a more reliable conclusion. Moreover, the results of studies mainly depend on the characteristics of patients included. Therefore, in this study, we aimed to determine whether baseline BMI affects clinical response to TCZ after six months of treatment in RA patients.

\section{PATIENTS AND METHODS}

In this single-center prospective cohort study, a total of 52 RA patients (10 males, 42 females; mean age $50.6 \pm 12.2$ years; range, 23 to 73 years) receiving $\mathrm{TCZ}$ were consecutively recruited and followed-up for six months at the department of rheumatology, Ningbo First Hospital between November 2013 and February 2017. All RA patients were diagnosed according to American Rheumatism Association 1987 revised criteria for the classification of $\mathrm{RA}^{17}$ or the 2010 American College of Rheumatology/European League Against Rheumatism (EULAR) criteria for RA. ${ }^{18}$ TCZ was given intravenously every four weeks at a usual dose of $8 \mathrm{mg} / \mathrm{kg}$, following corresponding recommendations. ${ }^{19}$ Increased or decreased doses of prednisone and conventional DMARDs (cDMARDs) were allowed at the discretion of the physician. The study protocol was approved by the Ningbo First Hospital Ethics Committee (Approval date: December $10^{\text {th }}, 2018$ ). A oral informed consent was obtained from each patient. The study was conducted in accordance with the principles of the Declaration of Helsinki.

The following data were collected at the start of TCZ treatment: age, sex, height, weight, disease duration, anti-cyclic citrullinated peptide antibody and rheumatoid factor status, health assessment questionnaire (HAQ) score, details of prior and concomitant use of cDMARDs and bDMARDs, and concomitant use of prednisone and corresponding doses. Additionally, values of the following variables were collected at baseline and month six: tender joint count and swollen joint count in 28 joints, patient global assessment and physician global assessment on visual analog scale $(0-100 \mathrm{~mm})$, and erythrocyte sedimentation rate (ESR). The disease activity score based on 28 joints (DAS28)-ESR and clinical disease activity index (CDAI) were calculated according to corresponding formulas. ${ }^{20,21}$ BMI was obtained by dividing weight in kilograms by the square of height in meters and was classified into three groups for Asian adults: normal $\left(\mathrm{BMI}<23.0 \mathrm{~kg} / \mathrm{m}^{2}\right)$, overweight $\left(23.0 \mathrm{~kg} / \mathrm{m}^{2} \leq \mathrm{BMI}<25.0 \mathrm{~kg} / \mathrm{m}^{2}\right)$, and obese $\left(\mathrm{BMI} \geq 25 \mathrm{~kg} / \mathrm{m}^{2}\right){ }^{22}$

The clinical response to $\mathrm{TCZ}$ was evaluated after six months. Since the acute phase reactants (such as ESR and CRP) were remarkably inhibited by TCZ, CDAI was suggested to be 


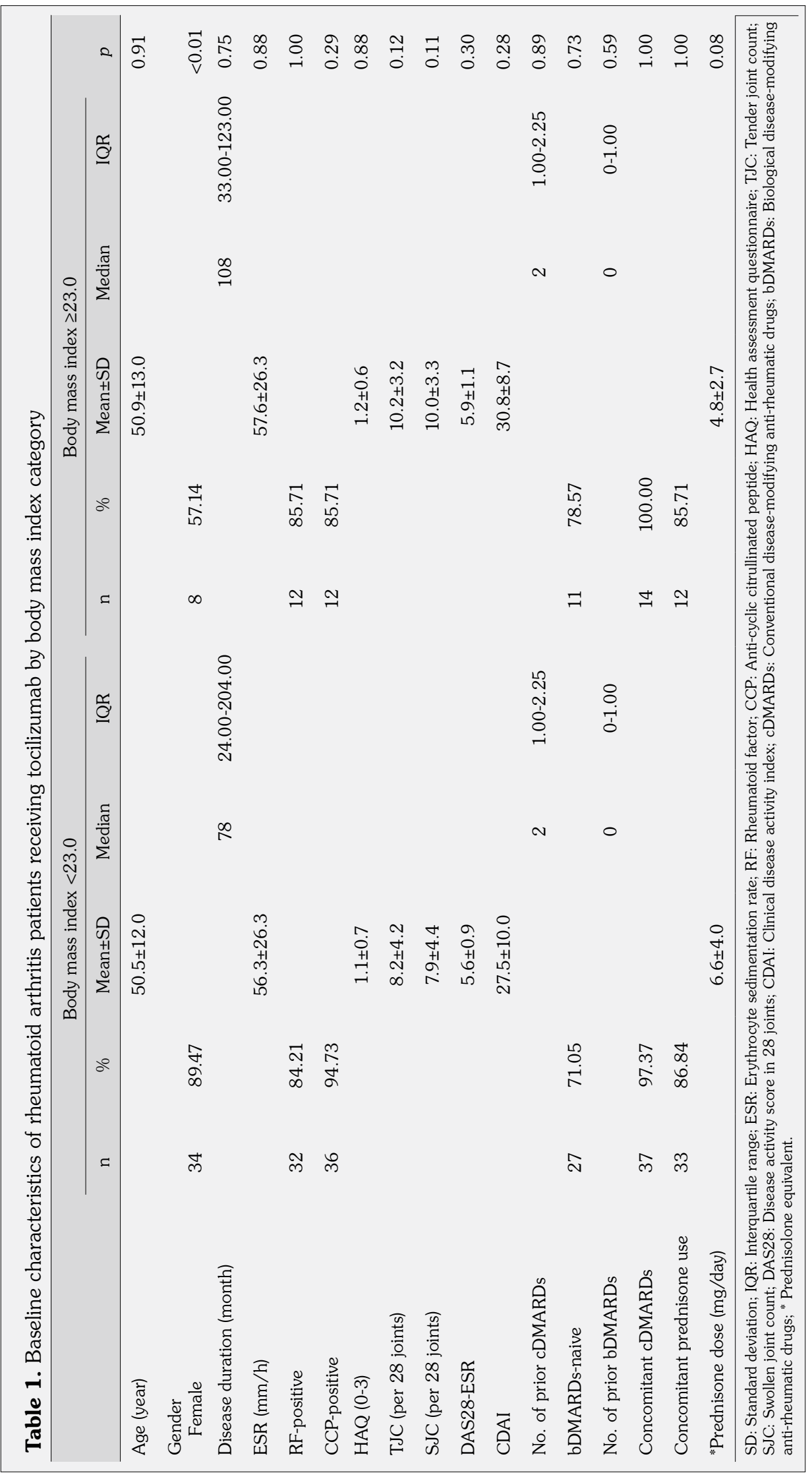


much more appropriate to evaluate the clinical response to TCZ compared with DAS28-ESR. ${ }^{23}$ Therefore, the primary clinical response criterion was CDAI low disease activity (LDA), which was defined as a $\mathrm{CDAI} \leq 10.0$. The secondary clinical response criteria included CDAI remission (CDAI $\leq 2.8$ ), DAS28-ESR LDA (DAS28-ESR $\leq 3.2)$, DAS28-ESR remission (DAS28-ESR $\leq 2.6$ ), EULAR good response, and decreased DAS28 $(\triangle \mathrm{DAS} 28-\mathrm{ESR}) \geq 1.2$. The EULAR good response was defined as a $\triangle \mathrm{DAS} 28-\mathrm{ESR} \geq 1.2$ and LDA (DAS28-ESR $\leq 3.2) .{ }^{24}$

\section{Statistical analysis}

Quantitative variables were expressed as mean (standard deviation) or median (interquartile range, IQR) according to the distribution of variable whether conformed to normal distribution. Categorical variables were expressed as absolute number and percentage (\%). Student's t-test and Mann-Whitney U test were employed for quantitative variables, and chi-square test or Fisher's exact test was applied for categorical variables. Regarding patients who withdrew before month six and in cases of missing data, the last observation carried forward method was applied. The relationship between $\mathrm{BMI}$ and clinical response to TCZ was assessed adopting the following two ways: the median level of BMI was compared between responders and non-responders, and the proportion of responders was compared between different BMI groups. All analysis was performed with PASW Statistics 18.0 software (SPSS, Inc., Chicago, IL, USA) and a two-tailed $p$ value less than 0.05 was considered statistically significant.

\section{RESULTS}

Among the 52 RA patients, 38 were bDMARDs-naive (73.1\%). Fifty-one patients (98.1\%) received concomitant cDMARDs and 45 patients $(86.5 \%)$ received concomitant prednisolone. The number of RA patients classified as normal weight, overweight, and obese according to BMI was $38(73.1 \%)$, eight (15.4\%), and six (11.5\%), respectively. The BMI median (IQR) for these RA patients was 20.81 $(19.15-23.61) \mathrm{kg} / \mathrm{m}^{2}$. Since the number of overweight and obese RA patients was relatively small, these two groups were combined. At the end of the six-month follow-up period, six patients discontinued the treatment: four due to lack of efficacy, one due to adverse events, and the other one due to economic reasons. As shown in Table 1, no significant evidence was found in the difference for baseline characteristics between normal weight and overweight/obese RA patients except for sex composition. Since non-significant difference was found in clinical response according to all assessment criteria used between males and females (all $p>0.05$ ), sex was not adjusted in the following analysis.

After six months of TCZ therapy, the number of RA patients achieving CDAI LDA was 39 (75.0\%). As shown in Table 2, similar baseline BMI median levels were found between RA patients reaching CDAI LDA and non-LDA: 21.11 (18.94-23.72) versus 20.78 (20.03-22.29) $(p=0.98)$. In addition, the number of RA patients reaching CDAI LDA among normal weight and overweight/obese RA patients was 27 (71.1\%) and 12 (85.7\%), respectively (Table 3). However,

Table 2. Body mass index level between responders and non-responders according to different clinical response criteria

\begin{tabular}{|c|c|c|c|c|c|c|}
\hline & \multicolumn{2}{|c|}{ Responders } & \multicolumn{2}{|c|}{ Non-responders } & \multirow[b]{2}{*}{ Z } & \multirow[b]{2}{*}{$p$} \\
\hline & Median & IQR & Median & IQR & & \\
\hline CDAI, LDA & 21.11 & $18.94-23.72$ & 20.78 & 20.03-22.29 & 0.02 & 0.98 \\
\hline $\mathrm{CDAI}$ remission & 21.10 & $18.84-23.68$ & 20.81 & $19.15-22.69$ & 0.05 & 0.96 \\
\hline DAS28-ESR, LDA & 20.83 & $19.29-23.72$ & 20.42 & $18.78-22.29$ & 0.78 & 0.43 \\
\hline DAS28-ESR remission & 20.83 & $19.29-23.72$ & 20.42 & $18.86-22.29$ & 0.71 & 0.48 \\
\hline EULAR good response & 20.83 & $19.29-23.72$ & 20.42 & $18.78-22.29$ & 0.78 & 0.43 \\
\hline$\Delta \mathrm{DAS} 28-\mathrm{ESR} \geq 1.2$ & 20.78 & $19.22-23.70$ & 21.11 & $18.37-22.31$ & 0.62 & 0.54 \\
\hline
\end{tabular}

IQR: Interquartile range; CDAI: Clinical disease activity index; LDA: Low disease activity; DAS28: Disease activity score in 28 joints; ESR: Erythrocyte sedimentation rate; EULAR: European League Against Rheumatism. 
Table 3. Proportion of responders among normal weight and overweight/obese rheumatoid arthritis patients according to different clinical response criteria

\begin{tabular}{|c|c|c|c|c|c|c|}
\hline \multirow[b]{2}{*}{ Groups } & \multicolumn{2}{|c|}{ Body mass index $<23.0$} & \multicolumn{2}{|c|}{ Body mass index $\geq 23.0$} & \multirow[b]{2}{*}{ Z } & \multirow[b]{2}{*}{$p$} \\
\hline & $\mathrm{n}$ & $\%$ & $\mathrm{n}$ & $\%$ & & \\
\hline \multicolumn{7}{|l|}{ CDAI LDA } \\
\hline Responders & 27 & 71.1 & 12 & 85.7 & NA & $0.47^{*}$ \\
\hline Non-responders & 11 & 28.9 & 2 & 14.3 & & \\
\hline \multicolumn{7}{|l|}{ CDAI remission } \\
\hline Responders & 12 & 31.6 & 6 & 42.9 & NA & $0.52^{*}$ \\
\hline Non-responders & 26 & 68.4 & 8 & 57.1 & & \\
\hline \multicolumn{7}{|l|}{ DAS28-ESR LDA } \\
\hline Responders & 21 & 55.3 & 10 & 71.4 & 1.11 & 0.29 \\
\hline Non-responders & 17 & 44.7 & 4 & 28.6 & & \\
\hline \multicolumn{7}{|c|}{ DAS28-ESR remission } \\
\hline Responders & 18 & 47.4 & 9 & 64.3 & 1.17 & 0.28 \\
\hline Non-responders & 20 & 52.6 & 5 & 15.7 & & \\
\hline \multicolumn{7}{|c|}{ EULAR good response } \\
\hline Responders & 21 & 55.3 & 10 & 71.4 & 1.11 & 0.29 \\
\hline Non-responders & 17 & 44.7 & 4 & 28.6 & & \\
\hline \multicolumn{7}{|l|}{$\Delta$ DAS28-ESR $\geq 1.2$} \\
\hline Responders & 32 & 84.2 & 13 & 92.9 & NA & $0.66^{*}$ \\
\hline Non-responders & 6 & 15.8 & 1 & 7.1 & & \\
\hline
\end{tabular}

non-significant evidence was found for difference in the proportion of responders between these two groups $(p=0.47)$.

After six months of TCZ therapy, 18 patients (34.6\%) with RA reached CDAI remission. When the baseline BMI median levels between RA patients reaching CDAI remission and non-remission was compared, non-significant difference was detected: 21.10 (18.84-23.68) versus 20.81 (19.15-22.69) ( $p=0.96)$ (Table 2). Similarly, non-significant difference was found in the proportion of patients achieving CDAI remission between normal weight (31.6\%) and overweight/obese (42.9\%) individuals $(p=0.52)$ (Table 3).

As shown in Tables 2 and 3, when the other clinical response criteria (DAS28-ESR LDA/DAS28-ESR remission/EULAR good response/ $\triangle \mathrm{DAS} 28-\mathrm{ESR} \geq 1.2$ ) were applied, no significant difference was found between BMI and clinical response to TCZ after six months of treatment.

\section{DISCUSSION}

Body mass index, a modifiable and easily recognized factor, has been reported to be associated with clinical response to IFX among RA patients in several studies. ${ }^{6-10}$ A clinically important question is whether BMI at treatment initiation also influences treatment response to other bDMARDs other than IFX, thus we conducted this study to determine the relationship between baseline BMI and clinical response to TCZ among RA patients using our prospective cohort study data. Although multiple clinical response criteria and different comparison methods (quantitative and qualitative comparison) have been utilized, non-significant evidence was found and our results are in accordance with the findings of previous studies. ${ }^{11-13}$

Body mass index, a simple and crude anthropometric measure that provides a marker of nutritional status, is usually applied to determine overweight and obesity in daily clinical practice and scientific research. Overweight 
and obesity are defined as the accumulation of abnormal or excessive fat within the body, and these conditions represent crucial risk factors associated with a variety of human diseases. ${ }^{25}$ In addition to providing vital space for energy storage, adipose tissue may also be regarded as an active endocrine organ and exert immune effects via producing pro-inflammatory cytokines and adipokines (the cytokines of adipocytes, such as leptin, adiponectin, resistin, and visfatin). ${ }^{14}$ Although the exact role of adipose tissue in RA remains unclear, emerging evidence suggests that there is a strong link between obesity/overweight and $\mathrm{RA}$, and the relationships between obesity/ overweight and different aspects of RA including the development, clinical parameters, and radiographic joint damage have been extensively examined. ${ }^{26}$ Although there have been conflicting reports on the impact of obesity on the risk of $\mathrm{RA}$, the majority of studies indicate a positive association, particularly in females, and a recent systematic review and meta-analysis indicates that overweight and obesity are significantly associated with the development of RA, showing that increased BMI could contribute to a higher risk for RA development. ${ }^{27}$ Similarly, the associations of obesity/overweight with clinical parameters (composite and individual disease activity measures, pain scores, and health assessment questionnaire scores) and radiographic joint damage have also been controversial, and a recent meta-analysis demonstrates that obesity is associated with increased DAS28 and HAQ score and with lower radiographic joint damage. ${ }^{28}$

In addition to the association of obesity/overweight with the development, clinical parameters, and radiographic progression damage of $\mathrm{RA}$, the relationship between obesity/overweight and clinical response to available DMARDs, particularly bDMARDs, has also attracted extensive attention. Initially, one study involving 89 RA patients treated by IFX intravenously found that baseline BMI was highly and negatively associated with the absolute decrease in the DAS28 after 16 weeks adjusted for the baseline DAS28 or anti-citrullinated protein antibody status. ${ }^{6}$ Subsequently, several studies have replicated the negative association of BMI at initial treatment with clinical response to IFX, RA patients with higher BMI having decreased likelihood to achieve favorable clinical response. ${ }^{7-10}$ Intriguingly, one study performed by Gremese et al. ${ }^{7}$ consisting of 641 patients with longstanding RA patients treated by three types of TNF- $\alpha$ inhibitors (adalimumab, etanercept, and IFX) found that obese patients were less likely to achieve remission compared with non-obese patients when all these three types of TNF- $\alpha$ inhibitors were considered. When data were analyzed according to the type of TNF- $\alpha$ inhibitor separately, significant evidence was only found for IFX, and the results indicated that obesity was particularly associated with clinical response to IFX.

Based on the finding of RA patients with higher BMI being less likely to respond well to IFX, ${ }^{6-10}$ it is natural to consider whether BMI affects the clinical response to bDMARDs other than IFX. As a consequence, the association between $\mathrm{BMI}$ and treatment response to abatacept (ABA, T-cell co-stimulation inhibitor), ${ }^{13,29-32}$ rituximab (B-cell depletion) ${ }^{33}$ and $\mathrm{TCZ}^{11,15,16}$ has been analyzed; however, non-significant evidence was found.

Tocilizumab is a recombinant humanized anti-IL-6 receptor monoclonal antibody which functions through blocking the biological effect of IL-6. However, non-significant difference was found in available studies. ${ }^{11,15,16}$ The first study concerning the relationship between BMI and treatment response to TCZ among RA patients was a multicentric retrospective study performed by Pers et al., ${ }^{11}$ in which a total of 222 patients receiving TCZ were included. However, non-significant difference was found in clinical response to TCZ in terms of EULAR response, DAS28 remission, and LDA at month six between overweight/obese and normal weight RA patients. ${ }^{11}$ Similarly, another multicenter retrospective study conducted in France, in which a total of 115 RA patients receiving $\mathrm{TCZ}$ were included, did not detect significant difference in treatment response after six months between overweight/obese and normal weight RA patients. ${ }^{15}$ Furthermore, a recent study performed in Korea included 68 RA patients treated by three types of bDMARDs, in which the number of patients treated by ABA, TCZ, and TNF- $\alpha$ inhibitor was 17, 24, and 27, respectively, and similar clinical response to bDMARDs as a whole or by each type was found between overweight/obese and 
normal weight RA patients. ${ }^{16}$ Taking our results into account, it might be concluded that BMI at initial treatment do not influence clinical response to TCZ among RA patients. In other words, TCZ may be an option for overweight/ obese RA patients compared with IFX. TCZ is administrated intravenously according to body weight and is a lipophilic drug that may be injected subcutaneously, suggesting that the pharmacokinetics of intravenous TCZ may not be altered by adipose tissue. ${ }^{15}$ This might account for the similar clinical response to TCZ between overweight/obese and normal weight RA patients.

Limitations of the present study should be mentioned. First, since the patients with RA were only recruited from our hospital and the majority of the patients were females and bDMARDsnaive, the selection bias was inevitable and the generalizability of our study might be limited. In addition, the sample size of this study is relatively small and the false negative result observed might be due to the relatively lower power caused by our small sample size. However, this might be due to the fact that the strength of association between BMI and clinical response might be weak, and the clinical value of $\mathrm{BMI}$ in guiding personalized treatment of TCZ for RA patients is limited. Nevertheless, the results of our study need further confirmation in studies with larger sample sizes.

In conclusion, data obtained from this prospective study indicate that BMI is not associated with clinical response to TCZ among RA patients and TCZ may be used to treat RA patients regardless of BMI levels.

\section{Declaration of conflicting interests}

The authors declared no conflicts of interest with respect to the authorship and/or publication of this article.

\section{Funding}

This work was supported by the National Natural Science Foundation of China (Grant No. 81602921), Nature Science Foundation of Ningbo city (Grant No. 2016A610159), Medical and Health Planned Science and Technology Project of Zhejiang province (Grant No. 2017KY582), Ningbo Scientific Innovation Team for Environmental Hazardous Factor Control and Prevention (Grant No. 2016C51001), and K.C. Wong Magna Fund in Ningbo University.

\section{REFERENCES}

1. Smolen JS, Aletaha D, McInnes IB. Rheumatoid arthritis. Lancet 2016;388:2023-38.

2. McInnes IB, Schett G. Pathogenetic insights from the treatment of rheumatoid arthritis. Lancet 2017 389:2328-37.

3. Smolen JS, Landewé R, Bijlsma J, Burmester G, Chatzidionysiou $\mathrm{K}$, Dougados $\mathrm{M}$, et al. EULAR recommendations for the management of rheumatoid arthritis with synthetic and biological diseasemodifying antirheumatic drugs: 2016 update. Ann Rheum Dis 2017;76:960-77.

4. Singh JA, Saag KG, Bridges SL Jr, Akl EA, Bannuru RR, Sullivan MC, et al. 2015 American College of Rheumatology Guideline for the Treatment of Rheumatoid Arthritis. Arthritis Rheumatol 2016;68:1-26.

5. Lau CS, Chia F, Harrison A, Hsieh TY, Jain R, Jung $\mathrm{SM}$, et al. APLAR rheumatoid arthritis treatment recommendations. Int $\mathrm{J}$ Rheum Dis 2015;18:685-713.

6. Klaasen R, Wijbrandts CA, Gerlag DM, Tak PP. Body mass index and clinical response to infliximab in rheumatoid arthritis. Arthritis Rheum 2011;63:359-64.

7. Gremese E, Carletto A, Padovan M, Atzeni F, Raffeiner B, Giardina AR, et al. Obesity and reduction of the response rate to anti-tumor necrosis factor $\alpha$ in rheumatoid arthritis: an approach to a personalized medicine. Arthritis Care Res (Hoboken) 2013;65:94-100.

8. Ottaviani S, Gardette A, Tubach F, Roy C, Palazzo $\mathrm{E}$, Gill $\mathrm{G}$, et al. Body mass index and response to infliximab in rheumatoid arthritis. Clin Exp Rheumatol 2015;33:478-83.

9. Heimans L, van den Broek M, le Cessie S, Siegerink B, Riyazi N, Han $\mathrm{KH}$, et al. Association of high body mass index with decreased treatment response to combination therapy in recent-onset rheumatoid arthritis patients. Arthritis Care Res (Hoboken) 2013;65:1235-42.

10. Levitsky A, Brismar K, Hafström I, Hambardzumyan $\mathrm{K}$, Lourdudoss $\mathrm{C}$, van Vollenhoven RF, et al. Obesity is a strong predictor of worse clinical outcomes and treatment responses in early rheumatoid arthritis: results from the SWEFOT trial. RMD Open 2017;3:e000458.

11. Pers YM, Godfrin-Valnet M, Lambert J, Fortunet C, Constant E, Mura T, et al. Response to tocilizumab in rheumatoid arthritis is not influenced by the body mass index of the patient. J Rheumatol 2015;42:580-4.

12. Wallenius V, Wallenius K, Ahrén B, Rudling M, Carlsten $\mathrm{H}$, Dickson SL, et al. Interleukin-6-deficient mice develop mature-onset obesity. Nat Med 2002;8:75-9.

13. Younis S, Rosner I, Rimar D, Boulman N, Rozenbaum M, Odeh $\mathrm{M}$, et al. Weight change during pharmacological blockade of interleukin- 6 or tumor necrosis factor- $\alpha$ in patients with inflammatory rheumatic disorders: a 16-week comparative study. Cytokine 2013;61:353-5. 
14. Abella V, Scotece M, Conde J, López V, Lazzaro V, Pino $\mathrm{J}$, et al. Adipokines, metabolic syndrome and rheumatic diseases. J Immunol Res 2014;2014:343746.

15. Gardette A, Ottaviani S, Sellam J, Berenbaum F, Lioté F, Meyer A, et al. Body mass index and response to tocilizumab in rheumatoid arthritis: a real life study. Clin Rheumatol 2016;35:857-61.

16. Kim SK, Choe JY, Park SH, Lee H. No predictive effect of body mass index on clinical response in patients with rheumatoid arthritis after 24 weeks of biological disease-modifying antirheumatic drugs: a single-center study. Clin Rheumatol 2016;35:1129-36.

17. Arnett FC, Edworthy SM, Bloch DA, McShane DJ, Fries JF, Cooper NS, et al. The American Rheumatism Association 1987 revised criteria for the classification of rheumatoid arthritis. Arthritis Rheum 1988;31:315-24.

18. Aletaha D, Neogi T, Silman AJ, Funovits J, Felson DT, Bingham CO, et al. 2010 Rheumatoid arthritis classification criteria: an American College of Rheumatology/European League Against Rheumatism collaborative initiative. Arthritis Rheum 2010;62:2569-81.

19. Exper group of tocilizumab in treating rheumatoid arthritis. Expert recommendations on tocilizumab in treating rheumatoid arthritis. Chin $\mathrm{J}$ Rheumatol 2013;17:436-8.

20. Prevoo ML, van 't Hof MA, Kuper $\mathrm{HH}$, van Leeuwen MA, van de Putte LB, van Riel PL. Modified disease activity scores that include twenty-eight-joint counts. Development and validation in a prospective longitudinal study of patients with rheumatoid arthritis. Arthritis Rheum 1995;38:44-8.

21. Aletaha D, Nell VP, Stamm T, Uffmann M, Pflugbeil $\mathrm{S}$, Machold $\mathrm{K}$, et al. Acute phase reactants add little to composite disease activity indices for rheumatoid arthritis: validation of a clinical activity score. Arthritis Res Ther 2005;7:796-806.

22. Shiwaku K, Anuurad E, Enkhmaa B, Nogi A, Kitajima $\mathrm{K}$, Shimono K, et al. Overweight Japanese with body mass indexes of 23.0-24.9 have higher risks for obesity-associated disorders: a comparison of Japanese and Mongolians. Int $\mathrm{J}$ Obes Relat Metab Disord 2004;28:152-8.

23. Kawashiri SY, Kawakami A, Iwamoto N, Fujikawa $\mathrm{K}$, Aramaki T, Tamai M, et al. Disease activity score 28 may overestimate the remission induction of rheumatoid arthritis patients treated with tocilizumab: comparison with the remission by the clinical disease activity index. Mod Rheumatol 2011;21:365-9.
24. van Gestel AM, Prevoo ML, van 't Hof MA, van Rijswijk $\mathrm{MH}$, van de Putte LB, van Riel PL. Development and validation of the European League Against Rheumatism response criteria for rheumatoid arthritis. Comparison with the preliminary American College of Rheumatology and the World Health Organization/ International League Against Rheumatism Criteria. Arthritis Rheum 1996;39:34-40.

25. Bischoff SC, Boirie Y, Cederholm T, Chourdakis M, Cuerda C, Delzenne NM, et al. Towards a multidisciplinary approach to understand and manage obesity and related diseases. Clin Nutr 2017;36:917-38.

26. Iannone F, Lopalco G, Rigante D, Orlando I, Cantarini L, Lapadula G. Impact of obesity on the clinical outcome of rheumatologic patients in biotherapy. Autoimmun Rev 2016;15:447-50.

27. Qin B, Yang M, Fu H, Ma N, Wei T, Tang Q, et al. Body mass index and the risk of rheumatoid arthritis: a systematic review and dose-response meta-analysis. Arthritis Res Ther 2015;17:86.

28. Vidal C, Barnetche T, Morel J, Combe B, Daïen C. Association of Body Mass Index Categories with Disease Activity and Radiographic Joint Damage in Rheumatoid Arthritis: A Systematic Review and Metaanalysis. J Rheumatol 2015;42:2261-9.

29. Gardette A, Ottaviani S, Sellam J, Berenbaum F, Lioté $\mathrm{F}$, Fautrel B, et al. Body mass index and response to abatacept in rheumatoid arthritis. Eur $\mathrm{J}$ Clin Invest 2016;46:1048-52.

30. Iannone F, Courvoisier DS, Gottenberg JE, Hernandez $\mathrm{MV}$, Lie E, Canhão $\mathrm{H}$, et al. Body mass does not impact the clinical response to intravenous abatacept in patients with rheumatoid arthritis. Analysis from the "pan-European registry collaboration for abatacept (PANABA). Clin Rheumatol 2017;36:773-9.

31. Mariette X, Alten R, Nüßlein HG, Galeazzi M, Lorenz HM, Cantagrel A, et al. The effect of body mass index on clinical response to abatacept as a first-line biologic for rheumatoid arthritis: 6-month results from the 2-year, observational, prospective ACTION study. Joint Bone Spine 2017;84:571-6.

32. D’Agostino MA, Alten R, Mysler E, Le Bars M, Ye $\mathrm{J}$, Murthy $\mathrm{B}$, et al. Body mass index and clinical response to intravenous or subcutaneous abatacept in patients with rheumatoid arthritis. Clin Rheumatol 2017;36:2655-65.

33. Ottaviani S, Gardette A, Roy C, Tubach F, Gill G, Palazzo E, et al. Body Mass Index and response to rituximab in rheumatoid arthritis. Joint Bone Spine 2015;82:432-6. 